\title{
New equalities connected with intuitionistic fuzzy matrices
}

\author{
D. Venkatesan ${ }^{1^{*}}$ and S. Sriram ${ }^{2}$
}

\begin{abstract}
This paper, introduce a new implication of intuitionistic fuzzy matrix and study its algebraic properties. Also investigates the equalities of intuitionistic fuzzy matrix in the case where this new implication is combined with the well known operations for intuitionistic fuzzy matrices.
\end{abstract}

\section{Keywords}

Intuitionistic Fuzzy Set(IFS), Intuitionistic Fuzzy Matrix(IFM), Intuitionistic Fuzzy Implication (IFI).

\section{AMS Subject Classification}

94D05, 15B15, 15B99.

${ }^{1}$ Department of Mathematics, Annamalai University Annamalainagar -608002, India.

${ }^{2}$ Mathematics wing, Directorate of Distance Education, Annamalai University, Annamalainagar -608002, India.

${ }^{*}$ Corresponding author: ${ }^{1}$ venkat2733327@gmail.com; ${ }^{2}$ ssm_3096@yahoo.co.in

Article History: Received 24 November 2018; Accepted 09 April 2019

\section{Contents}

1 Introduction ................................ 385

2 Preliminaries ............................. 385

3 Main Results . ............................ 386

4 New equalities connected with intuitionistic fuzzy matrices ..................................... 386

5

Conclusion .388

References 388

\section{Introduction}

In [1], he was introduced the concept of IFS as a generalization of fuzzy sets introduced by Zadeh [19]. From his the look, IFS is studied by many researchers and applies in many areas such as decision-making, clustering Analysis etc., Using the theory of fuzzy set, FMs as a generalization of matrices over the two elements Boolean algebra was studied by Kim and Roush [7]. Using the IFS theory, Im et al. [5] defined the idea of IFM as a generalization of the fuzzy matrix(FM).

In [12], they are established the set of all IFMs form a semiring with respect to the min-max composition of the IFM. shyamal and $\mathrm{Pal}[13,14]$, they are introduced two binary operations of FMs and established some of these properties and operations has been extended to IFMs and studied its algebraic properties by Sriram and Boobalan [3]. In [4], they are defined some types of IFMs, the min-max and max-min com- position of IFMs. Introduced a new composition operation and studied the algebraic properties also got a decomposition of an IFM by them [8]. In [9,10], they are studied representation and decomposition of an IFM using some $\left(\alpha, \alpha^{\prime}\right)$ cuts and reduction of an IFM to FM with some algebraic properties. Atanasov [2], he defined five new intuitionistic fuzzy operations on IFSs and taking multiplication and studied their algebraic properties. Verma and Sharma [15,16,17] developed some new results on IFSs and some new equalities connected with IFSs. In[18], we defined multiplicative operations of IFMs and investigated their algebraic properties.

The paper is organized as follows:

In Section 2 we shall briefly review IFMs and their operations.

In Section 3 Atanassov[1,2] defined a standard intuitionistic fuzzy implication for IFSs. We extend this implication to IFM and proved its algebraic properties. In Section 4 we formulate and prove some new elementary, but interesting equalities involving implication with other set operations on IFMs.

\section{Preliminaries}

This section briefly review IFMs and their operations.

Definition 2.1. : A fuzzy matrix is a matrix $A=\left(a_{i j}\right)$, where $a_{i j} \in[0,1]$.

Definition 2.2. ([6]): An IFM is a matrix of pairs 
$A=\left(\left\langle a_{i j}, a_{i j}^{\prime}\right\rangle\right)$ of a non negative real numbers satisfying $0 \leq a_{i j}+a_{i j}^{\prime} \leq 1$ for all $i, j$.

Definition 2.3. ([4]): For the IFMs $A$ and $B$ of same size, we have

(i) The max-min composition of $A$ and $B$ is defined by

$A \vee B=\left(\left\langle\max \left(a_{i j}, b_{i j}\right), \min \left(a_{i j}^{\prime}, b_{i j}^{\prime}\right)\right\rangle\right)$.

(ii) The min-max composition of $A$ and $B$ is defined by

$A \wedge B=\left(\left\langle\min \left(a_{i j}, b_{i j}\right), \max \left(a_{i j}^{\prime}, b_{i j}^{\prime}\right)\right\rangle\right)$.

(iii) The complement of a IFM A is $A^{C}=\left(\left\langle a_{i j}^{\prime}, a_{i j}\right\rangle\right)$.

Definition 2.4. ([4]): For the IFMs $A$ and $B$ of same size, $A \geq B$ if and only if $a_{i j} \geq b_{i j}$ and $a_{i j}^{\prime} \leq b_{i j}^{\prime}$ for all $i, j$.

Definition 2.5. ([4]): Let $O$ be an $m \times n$ zero IFM that have all entries are $\langle 0,1\rangle$.

Let $J$ be an $m \times n$ universal IFM $J$ is an IFM that have all entries are $\langle 1,0\rangle$.

Definition 2.6. ([18]): For the IFMs $A$ and $B$ of same size, then we have

(i) $A X_{1} B=\left(\left\langle\max \left(a_{i j}, b_{i j}\right), a_{i j}^{\prime} b_{i j}^{\prime}\right\rangle\right)$.

(ii) $A X_{2} B=\left(\left\langle a_{i j} b_{i j}, \max \left(a_{i j}^{\prime}, b_{i j}^{\prime}\right)\right\rangle\right)$.

Where'.' is the ordinary multiplication.

\section{Main Results}

Atanassov [1,2] defined a standard intuitionistic fuzzy implication for IFSs. Verma and Sharma[15, 16,17] proved the results associated with this implication for IFSs. We extend this implication of IFSs to IFMs and proved its algebraic properties.

Definition 3.1. For the IFMs $A$ and $B$ of same size, then we define $A \rightarrow_{1} B=\left(\left\langle\max \left(a_{i j}^{\prime}, b_{i j}\right), \min \left(a_{i j}, b_{i j}^{\prime}\right)\right\rangle\right)$.

This implication is constracted in such way that they produces an IFM

$$
\begin{aligned}
0 & \leq \max \left(a_{i j}^{\prime}, b_{i j}\right)+\min \left(a_{i j}, b_{i j}^{\prime}\right) \\
& \leq \max \left(a_{i j}^{\prime}, b_{i j}\right)+\min \left(1-a_{i j}^{\prime}, 1-b_{i j}\right) \\
& \leq \max \left(a_{i j}^{\prime}, b_{i j}\right)+1-\max \left(a_{i j}^{\prime}, b_{i j}\right) \\
& \leq 1 .
\end{aligned}
$$

Proposition 3.2. For the zero IFM $O$ and universal IFM J of same size, we have

(i) $O \rightarrow_{1} O=J$.

(ii) $O \rightarrow_{1} J=J$.

(iii) $J \rightarrow_{1} O=O$.

(iv) $J \rightarrow_{1} J=J$.

Proof. $(i) O \rightarrow{ }_{1} O$

$$
\begin{aligned}
& =(\langle\max (1,0), \min (0,1)\rangle) \\
& =(\langle 1,0\rangle) \\
& =J
\end{aligned}
$$

Hence, $O \rightarrow_{1} O=J$.

The proof (ii), (iii) and (iv) are similar to that of (i).

Proposition 3.3. For the IFMs A and B of same size, we have

(i) $A \rightarrow{ }_{1} A^{C}=A^{C}$.

(ii) $A \rightarrow_{1} B=B^{C} \rightarrow_{1} A^{C}$.

Proof. (i) $A \rightarrow{ }_{1} A^{C}$

$$
\begin{aligned}
& =\left(\left\langle\max \left(a_{i j}^{\prime}, a_{i j}^{\prime}\right), \min \left(a_{i j}, a_{i j}\right)\right\rangle\right) \\
& =\left(\left\langle a_{i j}^{\prime}, a_{i j}\right\rangle\right) \\
& =A^{C} .
\end{aligned}
$$

Hence, $A \rightarrow{ }_{1} A^{C}=A^{C}$.

(ii) $A \rightarrow{ }_{1} B$

$$
\begin{aligned}
& =\left(\left\langle\max \left(a_{i j}^{\prime}, b_{i j}\right), \min \left(a_{i j}, b_{i j}^{\prime}\right)\right\rangle\right) \\
& =B^{C} \rightarrow_{1} A^{C} .
\end{aligned}
$$

Hence, $A \rightarrow_{1} B=B^{C} \rightarrow_{1} A^{C}$.

Proposition 3.4. For any IFM A, we have

(i) $A \rightarrow_{1} J=J$.

(ii) $J \rightarrow_{1} A=A$.

(iii) $O \rightarrow_{1} A=J$.

(iv) $A \rightarrow_{1} O=A^{C}$.

Proof. Let $A=\left(\left\langle a_{i j}, a_{i j}^{\prime}\right\rangle\right)$ be an IFM. Then (i) $A \rightarrow{ }_{1} J$

$$
\begin{aligned}
& =\left(\left\langle\max \left(a_{i j}^{\prime}, 1\right), \min \left(a_{i j}, 0\right)\right\rangle\right) \\
& =(\langle 1,0\rangle) \\
& =J .
\end{aligned}
$$

Hence, $A \rightarrow_{1} J=J$.

The proof (ii),(iii) and (iv) are similar to that of (i).

\section{New equalities connected with intuitionistic fuzzy matrices}

In this section, we formulate and prove some new elementary, but interesting equalities involving implication with other set operations on IFMs.

Proposition 4.1. For the IFMs A and B of same size, we have

(i) $\left(A^{C} \rightarrow_{1} B\right) X_{1}\left(A \rightarrow{ }_{1} B^{C}\right)^{C}=A X_{1} B$.

(ii) $\left(A^{C} \rightarrow_{1} B\right) X_{2}\left(A \rightarrow_{1} B^{C}\right)^{C}=A X_{2} B$.

(iii) $\left(A \rightarrow_{1} B\right)^{C} X_{1}\left(B \rightarrow_{1} A\right)=A X_{1} B^{C}$.

(iv) $\left(A \rightarrow_{1} B\right)^{C} X_{2}\left(B \rightarrow_{1} A\right)=A X_{2} B^{C}$.

(v) $\left(A X_{1} B\right)^{C} \rightarrow_{1}\left(A X_{2} B\right)=A X_{1} B$.

(vi) $\left(A X_{2} B\right)^{C} \rightarrow_{1}\left(A X_{1} B\right)=A X_{1} B$.

(vii) $\left(A X_{1} B\right)^{C} \rightarrow_{1}(A @ B)=A X_{1} B$.

(viii) $\left(A X_{2} B\right)^{C} \rightarrow_{1}(A @ B)=A @ B$.

$(i x)(A @ B)^{C} \rightarrow_{1}\left(A X_{1} B\right)=A X_{1} B$.

$(x)(A @ B)^{C} \rightarrow_{1}\left(A X_{2} B\right)=A @ B$. 
Proof. $(i)\left(A^{C} \rightarrow_{1} B\right) X_{1}\left(A \rightarrow_{1} B^{C}\right)^{C}$

$$
\begin{aligned}
= & \left(\left\langle\max \left(a_{i j}, b_{i j}\right), \min \left(a_{i j}^{\prime}, b_{i j}^{\prime}\right)\right\rangle\right) X_{1} \\
& \left(\left\langle\min \left(a_{i j}, b_{i j}\right), \max \left(a_{i j}^{\prime}, b_{i j}^{\prime}\right)\right\rangle\right) \\
= & \left(\operatorname { m a x } \left(\max \left(a_{i j}, b_{i j}\right), \min \left(a_{i j}, b_{i j}\right),\right.\right. \\
& \left.\min \left(a_{i j}^{\prime}, b_{i j}^{\prime}\right) \max \left(a_{i j}^{\prime}, b_{i j}^{\prime}\right)\right) \\
= & \left(\left\langle\max \left(a_{i j}, b_{i j}\right), a_{i j}^{\prime} b_{i j}^{\prime}\right\rangle\right) \\
= & A X_{1} B .
\end{aligned}
$$

Hence, $\left(A^{C} \rightarrow_{1} B\right) X_{1}\left(A \rightarrow_{1} B^{C}\right)^{C}=A X_{1} B$.

The proof (ii),(iii),(iv),(v),(vi),(vii),(viii),(ix) and (x) are similar to that of (i).

Proposition 4.2. For the IFMs A and B of same size, we have

$$
\begin{aligned}
& (i)\left(\left(A X_{1} B\right) \rightarrow_{1}\left(A X_{2} B\right)^{C}\right)^{C}=A X_{2} B . \\
& (i i)\left(\left(A X_{2} B\right) \rightarrow_{1}\left(A X_{1} B\right)^{C}\right)^{C}=A X_{2} B . \\
& (i i i)\left(\left(A X_{1} B\right) \rightarrow_{1}(A @ B)^{C}\right)^{C}=A @ B . \\
& (\text { iv })\left((A @ B) \rightarrow_{1}\left(A X_{1} B\right)^{C}\right)^{C}=A @ B . \\
& (v)\left(\left(A X_{2} B\right) \rightarrow_{1}(A @ B)^{C}\right)^{C}=A X_{2} B . \\
& (v i)\left((A @ B) \rightarrow_{1}\left(A X_{2} B\right)^{C}\right)^{C}=A X_{2} B .
\end{aligned}
$$

Proof. (i) $\left(A X_{1} B\right) \rightarrow_{1}\left(A X_{2} B\right)^{C}$

$$
\begin{gathered}
=\left(\left\langle\max \left(a_{i j}, b_{i j}\right), a_{i j}^{\prime} b_{i j}^{\prime}\right\rangle\right) \rightarrow_{1} \\
\left(\left\langle\max \left(a_{i j}^{\prime}, b_{i j}^{\prime}\right), a_{i j} b_{i j}\right\rangle\right) \\
=\left(\max \left(a_{i j}^{\prime} b_{i j}^{\prime}, \max \left(a_{i j}^{\prime}, b_{i j}^{\prime}\right)\right),\right. \\
\left.\min \left(\max \left(a_{i j}, b_{i j}\right), a_{i j} b_{i j}\right)\right) \\
\left.=\left(\left\langle\max \left(a_{i j}^{\prime}, b_{i j}^{\prime}\right), a_{i j} b_{i j}\right\rangle\right)\right) \\
\left(\left(A X_{1} B\right) \rightarrow{ }_{1}\left(A X_{2} B\right)^{C}\right)^{C} \\
=\left(\left\langle a_{i j} b_{i j}, \max \left(a_{i j}^{\prime}, b_{i j}^{\prime}\right)\right\rangle\right) \\
=A X_{2} B .
\end{gathered}
$$

Hence, $\left(\left(A X_{1} B\right) \rightarrow_{1}\left(A X_{2} B\right)^{C}\right)^{C}=A X_{2} B$.

The proof (ii),(iii),(iv),(v) and (vi) are similar to that of (i).

Proposition 4.3. For the IFMs $A$ and $B$ of same size, we have

$$
\begin{aligned}
& (i)\left(\left(A^{C} \rightarrow_{1} B\right) X_{1}\left(A \rightarrow_{1} B^{C}\right)^{C}\right) \vee \\
& \left(\left(A^{C} \rightarrow_{1} B\right) X_{2}\left(A \rightarrow_{1} B^{C}\right)^{C}\right)=A X_{1} B . \\
& \text { (ii) }\left(\left(A^{C} \rightarrow_{1} B\right) X_{1}\left(A \rightarrow_{1} B^{C}\right)^{C}\right) \wedge \\
& \left(\left(A^{C} \rightarrow_{1} B\right) X_{2}\left(A \rightarrow_{1} B^{C}\right)^{C}\right)=A X_{2} B . \\
& \text { (iii) }\left(\left(A \rightarrow_{1} B\right)^{C} X_{1}\left(B \rightarrow_{1} A\right)\right) \vee \\
& \left(\left(A \rightarrow_{1} B\right)^{C} X_{2}\left(B \rightarrow_{1} A\right)\right)=A X_{1} B^{C} . \\
& \text { (iv) }\left(\left(A \rightarrow_{1} B\right)^{C} X_{1}\left(B \rightarrow_{1} A\right)\right) \wedge \\
& \left(\left(A \rightarrow_{1} B\right)^{C} X_{2}\left(B \rightarrow_{1} A\right)\right)=A X_{2} B^{C} . \\
& (v)\left(\left(A X_{1} B\right)^{C} \rightarrow_{1}\left(A X_{2} B\right)\right) \vee\left(\left(A X_{2} B\right)^{C} \rightarrow_{1}\left(A X_{1} B\right)\right) \\
& =A X_{1} B \text {. } \\
& (v i)\left(\left(A X_{1} B\right)^{C} \rightarrow_{1}\left(A X_{2} B\right)\right) \wedge\left(\left(A X_{2} B\right)^{C} \rightarrow_{1}\left(A X_{1} B\right)\right) \\
& =A X_{1} B \text {. } \\
& (v i i)\left(\left(A X_{1} B\right)^{C} \rightarrow_{1}(A @ B)\right) \vee\left(\left(A X_{2} B\right)^{C} \rightarrow_{1}(A @ B)\right) \\
& =A X_{1} B \text {. } \\
& \left(\text { viii) }\left(\left(A X_{1} B\right)^{C} \rightarrow_{1}(A @ B)\right) \wedge\left(\left(A X_{2} B\right)^{C} \rightarrow_{1}(A @ B)\right)\right. \\
& =A @ B \text {. } \\
& (i x)\left((A @ B)^{C} \rightarrow_{1}\left(A X_{1} B\right)\right) \vee\left((A @ B)^{C} \rightarrow_{1}\left(A X_{2} B\right)\right) \\
& =A X_{1} B \text {. }
\end{aligned}
$$

$$
\begin{aligned}
& (x)\left((A @ B)^{C} \rightarrow_{1}\left(A X_{1} B\right)\right) \wedge\left((A @ B)^{C} \rightarrow_{1}\left(A X_{2} B\right)\right) \\
& =A @ B . \\
& \text { Proof. }(i)\left(\left(A^{C} \rightarrow_{1} B\right) X_{1}\left(A \rightarrow_{1} B^{C}\right)^{C}\right) \vee \\
& \left(\left(A^{C} \rightarrow_{1} B\right) X_{2}\left(A \rightarrow_{1} B^{C}\right)^{C}\right) \\
& =\left(\left(\max \left(a_{i j}, b_{i j}\right), \min \left(a_{i j}^{\prime}, b_{i j}^{\prime}\right)\right) X_{1}\right. \\
& \left.\left(\min \left(a_{i j}, b_{i j}\right), \max \left(a_{i j}^{\prime}, b_{i j}^{\prime}\right)\right)\right) \vee \\
& \left(\left(\max \left(a_{i j}, b_{i j}\right), \min \left(a_{i j}^{\prime}, b_{i j}^{\prime}\right)\right) X_{2}\right. \\
& \left.\left(\min \left(a_{i j}, b_{i j}\right), \max \left(a_{i j}^{\prime}, b_{i j}^{\prime}\right)\right)\right) \\
& =\left(\max \left(\max \left(a_{i j}, b_{i j}\right), \min \left(a_{i j}, b_{i j}\right)\right),\right. \\
& \left.\min \left(a_{i j}^{\prime}, b_{i j}^{\prime}\right) \max \left(a_{i j}^{\prime}, b_{i j}^{\prime}\right)\right) \vee \\
& \left(\max \left(a_{i j}, b_{i j}\right) \min \left(a_{i j}, b_{i j}\right)\right. \text {, } \\
& \left.\max \left(\min \left(a_{i j}^{\prime}, b_{i j}^{\prime}\right), \max \left(a_{i j}^{\prime}, b_{i j}^{\prime}\right)\right)\right) \\
& =\left(\left\langle\max \left(a_{i j}, b_{i j}\right), a_{i j}^{\prime} b_{i j}^{\prime}\right\rangle\right) \vee \\
& \left(\left\langle a_{i j} b_{i j}, \max \left(a_{i j}^{\prime}, b_{i j}^{\prime}\right)\right\rangle\right) \\
& =\left(\max \left(\max \left(a_{i j}, b_{i j}\right), a_{i j} b_{i j}\right)\right. \text {, } \\
& \left.\min \left(a_{i j}^{\prime} b_{i j}^{\prime}, \max \left(a_{i j}^{\prime}, b_{i j}^{\prime}\right)\right)\right) \\
& =\left(\left\langle\max \left(a_{i j}, b_{i j}\right), a_{i j}^{\prime} b_{i j}^{\prime}\right\rangle\right) \\
& =A X_{1} B \text {. }
\end{aligned}
$$

Hence, $\left(A^{C} \rightarrow_{1} B\right) X_{1}\left(A \rightarrow_{1} B^{C}\right)^{C} \vee$

$$
\left(A^{C} \rightarrow_{1} B\right) X_{2}\left(A \rightarrow{ }_{1} B^{C}\right)^{C}=A X_{1} B .
$$

The proof (ii),(iii),(iv),(v),(vi),(vii),(viii),(ix) and (x) are similar to that of (i).

Proposition 4.4. For the IFMs A and B of same size, we have

$$
\begin{aligned}
& \text { (i) }\left(\left(\left(A X_{1} B\right) \rightarrow_{1}\left(A X_{2} B\right)^{C}\right)^{C}\right) \vee\left(\left(\left(A X_{2} B\right) \rightarrow_{1}\left(A X_{1} B\right)^{C}\right)^{C}\right) \\
& =A X_{2} B \text {. } \\
& \text { (ii) }\left(\left(\left(A X_{1} B\right) \rightarrow_{1}\left(A X_{2} B\right)^{C}\right)^{C}\right) \wedge\left(\left(\left(A X_{2} B\right) \rightarrow_{1}\left(A X_{1} B\right)^{C}\right)^{C}\right) \\
& =A X_{2} B \text {. } \\
& (\text { iii })\left(\left(A X_{1} B\right) \rightarrow_{1}(A @ B)^{C}\right)^{C} \vee\left((A @ B) \rightarrow_{1}\left(A X_{1} B\right)^{C}\right)^{C} \\
& =A @ B . \\
& (\text { iv })\left(\left(A X_{1} B\right) \rightarrow_{1}(A @ B)^{C}\right)^{C} \wedge\left((A @ B) \rightarrow_{1}\left(A X_{1} B\right)^{C}\right)^{C} \\
& =A @ B \text {. } \\
& (v)\left(\left(A X_{2} B\right) \rightarrow_{1}(A @ B)^{C}\right)^{C} \vee\left((A @ B) \rightarrow_{1}\left(A X_{2} B\right)^{C}\right)^{C} \\
& =A X_{2} B \text {. } \\
& (v i)\left(\left(A X_{2} B\right) \rightarrow_{1}(A @ B)^{C}\right)^{C} \wedge\left((A @ B) \rightarrow_{1}\left(A X_{2} B\right)^{C}\right)^{C} \\
& =A X_{2} B \text {. } \\
& \left(\left(\left(A X_{2} B\right) \rightarrow_{1}\left(A X_{1} B\right)^{C}\right)^{C}\right) \\
& =\left(\left(\max \left(a_{i j}, b_{i j}\right), a_{i j}^{\prime} b_{i j}^{\prime}\right) \rightarrow_{1}\right. \\
& \left.\left(\max \left(a_{i j}^{\prime}, b_{i j}^{\prime}\right), a_{i j} b_{i j}\right)\right) \\
& \vee\left(\left(a_{i j} b_{i j}, \max \left(a_{i j}^{\prime}, b_{i j}^{\prime}\right)\right) \rightarrow_{1}\right. \\
& \left.\left(a_{i j}^{\prime} b_{i j}^{\prime}, \max \left(a_{i j}, b_{i j}\right)\right)\right) \\
& =\left(\max \left(a_{i j}^{\prime} b_{i j}^{\prime}, \max \left(a_{i j}^{\prime}, b_{i j}^{\prime}\right)\right),\right. \\
& \min \left(\max \left(a_{i j}, b_{i j}\right), a_{i j} b_{i j}\right) \vee \\
& \left(\max \left(\max \left(a_{i j}^{\prime}, b_{i j}^{\prime}\right), a_{i j}^{\prime} b_{i j}^{\prime}\right)\right. \text {, } \\
& \left.\min \left(a_{i j} b_{i j}, \max \left(a_{i j}, b_{i j}\right)\right)\right) \\
& =\left(\left(\max \left(a_{i j}^{\prime}, b_{i j}^{\prime}\right), a_{i j} b_{i j}\right) \vee\left(\max \left(a_{i j}^{\prime}, b_{i j}^{\prime}\right), a_{i j} b_{i j}\right)\right) \\
& =\left(a_{i j} b_{i j}, \max \left(a_{i j}^{\prime}, b_{i j}^{\prime}\right)\right) \vee\left(a_{i j} b_{i j}, \max \left(a_{i j}^{\prime}, b_{i j}^{\prime}\right)\right) \\
& =\left(\max \left(a_{i j} b_{i j}, a_{i j} b_{i j}\right)\right. \text {, } \\
& \left.\min \left(\max \left(a_{i j}^{\prime}, b_{i j}^{\prime}\right), \max \left(a_{i j}^{\prime}, b_{i j}^{\prime}\right)\right)\right) \\
& =\left(\left\langle a_{i j} b_{i j}, \max \left(a_{i j}^{\prime}, b_{i j}^{\prime}\right)\right\rangle\right)
\end{aligned}
$$




$$
\begin{gathered}
=A X_{2} B . \\
\text { Hence, }\left(\left(\left(A X_{1} B\right) \rightarrow_{1}\left(A X_{2} B\right)^{C}\right)^{C}\right) \vee\left(\left(\left(A X_{2} B\right)\right.\right. \\
\left.\left.\quad \rightarrow_{1}\left(A X_{1} B\right)^{C}\right)^{C}\right)=A X_{2} B .
\end{gathered}
$$

The proof (ii),(iii),(iv),(v) and (vi) are similar that of (i).

\section{Conclusion}

This paper, we have defined an intuitionistic fuzzy implication for intuitionistic fuzzy matrices and proved its algebraic properties. Also elementary equalities involving this implication with well known operations of IFMs are inverstigated.

\section{References}

[1] K. Atanassov, Intuitionistic Fuzzy Set, Fuzzy Sets and System, 20(1)(1983), 87-96.

[2] K. Atanassov, E. Szmidt and J. Kacprzyk, Intuitionistic fuzzy implication $\rightarrow 187$,Notes on Intuitionistic Fuzzy Sets, 23(2)(2017), 37-43.

[3] J. Boobalan and S. Sriram, Arithmetic operations on Intuitionistic Fuzzy Matrices, Proceedings of the International Conference On Mathematical Sciences, Sathyabama University, (2014), 484-487.

[4] E.G. Emam and M. A. Fndh, Some results associated wiith the max-min and min-max compositions of bifuzzy matrices, Journal of the Egyption Mathematical Society, (2016), 515-521.

[5] Y. B. Im, E.B. Lee and S. W. Park, The Determinant of square Intuitionistic Fuzzy Matrices, Far East Journal of Mathematical Sciences, 3(5)(2001), 789-796.

[6] S. K. Khan, M. Pal and A. K. Shyamal, Intuitionistic Fuzzy Matrices, Notes on Intuitionistic Fuzzy Sets, 8(2)(2002), 51-62.

[7] K. H. Kim and R. W. Roush, Generalized fuzzy matrices, Fuzzy Sets and Systems, 4(1980), 293-315.

[8] T. Muththuraji, S. Sriram and P. Murugadas, Decomposition of Intuitionistic Fuzzy Matrices, Fuzzy Information and Engineering, 8(2016), 345-354.

[9] T. Muththuraji and S.Sriram, Representation and decomposition of an intuitionistic fuzzy matrix using some $\left(\alpha, \alpha^{\prime}\right)$ cuts, Appl.Appl.Math., 1(12)(2017), 241-258.

[10] T. Muththuraji and S. Sriram, Reduction of an intuitionistic fuzzy matrix to fuzzy matrix with some algebraic properties, Annals of Fuzzy Mathematics and Informatics, 13(4)(2017), 475-483.

[11] M. Pal, Intuitionistic fuzzy determinant, V.U.J. Physical Sciences, 7(2001), 87-93.

[12] S. Sriram and P. Murugadas, On Semi-ring of Intuitionistic Fuzzy Matrices, Applied Mathematical Science, 4(23)(2010), 1099-1105.

[13] A. K. Shyamal and M. Pal, Distances between intuitionistic fuzzy matrices, V.U.J. Physical Sciences, 8(2002), 81-91.

[14] A. K. Shyamal and M. Pal, Two new operators on fuzzy matrices, J. Applied Mathematics and Computation, 15(2004), 91-107.
[15] R. K. Verma, and B. D. Sharma, Some new results on Intuitionistic Fuzzy Sets, Proceedings of the jangjeon Mathematical Society, 16(1)(2013), 101-114.

[16] R. K. Verma, and B. D. Sharma, Intuitionistic Fuzzy Sets: Some new results, Notes on Intuitionistic Fuzzy Sets, 17(3)(2011), 1-10.

[17] R. K. Verma, and B. D. Sharma, Some new equalities connected with, Intuitionistic Fuzzy Sets, Notes on Intuitionistic Fuzzy Sets, 19(2)(2013), 25-30.

[18] D. Venkatesan and S. Sriram, Multiplicative operations of Intuitionistic Fuzzy Matrices,Annals of Pure and Applied Mathematics, 14(1)(2017), 173-181.

[19] L. A. Zadeh, Fuzzy Sets, Journal of Information and Control, 8(1965), 338-353.



$$
\begin{aligned}
& \text { ISSN(P):2319 - } 3786 \\
& \text { Malaya Journal of Matematik } \\
& \text { ISSN(O):2321 - } 5666
\end{aligned}
$$

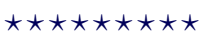

